\title{
PREDICTION OF THE CORROSION CRACKING OF STRUCTURES UNDER THE CONDITIONS OF HIGH-TEMPERATURE CREEP
}

\author{
O. K. Morachkovskii ${ }^{1,2}$ and Yu. V. Romashov ${ }^{1}$
}

UDC 539.3

\begin{abstract}
On the basis of the continual model of corrosion crack growth proposed earlier and the well-known incremental-type creep theory, we make an attempt to predict the corrosion cracking of structures under the conditions of high-temperature creep. We propose the mathematical statement of the problem taking into account the influence on corrosion cracking of the properties of corrosive media and the redistribution of stresses in time caused by creep. The Bubnov-Galerkin method is applied for the solution of this problem. An example of prediction of the phenomenon of corrosion cracking in the case of creep of a pipe under the action of internal pressure is analyzed.
\end{abstract}

Keywords: corrosion cracking, creep, damageability, Bubnov-Galerkin method, pipe under the action of internal pressure.

The urgency of the problem of prediction of corrosion cracking under the conditions of high-temperature creep is explained by the fact that structural elements subjected to corrosion are, as a rule, costly, irreparable, and difficultly replaced. Among elements of this sort, we can mention, e.g., the superheaters of steam boilers [1]. Creep leads to inhomogeneous and continuously varying (with time) changes in the shape of structural elements accompanied by the redistribution of stresses and damage. The influence of these processes on the corrosion cracking of structural elements depends on the properties of their materials, external loads, the geometric shape of the elements, and the type of fastening. The analysis of these factors can be performed on the basis of the mechanics of deformable solids and the regularities of behavior of the materials under the conditions of corrosion cracking and creep. Thus, this approach was applied in [2] to the solution of the problem of evaluation of the period of subcritical crack growth under the conditions of high-temperature creep for a wheel of the steam turbine. The aim of the present work is to predict corrosion cracking under the conditions of high-temperature creep on the basis of the continual model of corrosion crack growth proposed in [3] and the incremental-type theory of creep.

\section{Mathematical Statement of the Problem}

In a Cartesian coordinate system $x_{k}, k=1,2,3$, we consider a deformed solid body with volume $\Upsilon$ and surface $v$ loaded by a given volume forces $f_{i}$ and surface forces $p_{i}$ (applied to a part of the surface $v_{p} \subset v$ ). On the restrained part of the surface $v_{u} \subset v$, we specify the displacements of points of the body $u_{i}^{(0)}$. In addition, it is assumed that the body interacts with a medium through a part of the surface $v_{S C C} \subset v$, and this

\footnotetext{
1 "Kharkov Polytechnic Institute” National Technical University, Kharkov, Ukraine.

${ }^{2}$ Corresponding author; e-mail: morachko@kpi.kharkov.ua.
}

Translated from Fizyko-Khimichna Mekhanika Materialiv, Vol.46, No.5, pp.43-47, September-October, 2010. Original article submitted September 27, 2010. 
interaction leads to the corrosion cracking of the body. The stationary thermal state of the body is assumed to be given and determined by the temperature field $T=T\left(x_{k}\right), x_{k} \in \Upsilon$. The state of the body deformed under the conditions of creep at any time $t \geq 0$ is characterized by the components of the vector of displacements $u_{i}=$ $u_{i}\left(t, x_{k}\right)$, stress tensor $\sigma_{i j}=\sigma_{i j}\left(t, x_{i}\right)$, total strain tensor $\varepsilon_{i j}=\varepsilon_{i j}\left(t, x_{i}\right)$, and creep strain tensor $c_{i j}=$ $c_{i j}\left(t, x_{k}\right)$, and the degree of damage $\omega_{c}=\omega_{c}\left(t, x_{k}\right)$ [4]. To describe the process of corrosion crack growth, we use [3] the parameter of corrosion cracking $\omega_{S C C}=\omega_{S C C}\left(t, x_{k}\right), 0 \leq \omega_{S C C}<1, x_{k} \in v_{S C C}$, corresponding to the normalized length of visually undetectable cracks. Then the complete system of equations aimed at the description of creep and corrosion cracking of homogeneous isotropic deformed solid bodies for low strains can be represented in the following form:

$$
\begin{gathered}
\frac{\partial \sigma_{i j}}{\partial x_{j}}+f_{i}=0, \quad \sigma_{i j} n_{j}=p_{i}, \quad x_{k} \in v_{p}, \\
\sigma_{i j} n_{i} n_{j}=-p_{S C C}, \quad \sigma_{i j} \tau_{i} n_{j}=0, \quad \sigma_{i j} b_{i} n_{j}=0, \quad x_{k} \in v_{S C C}, \\
\varepsilon_{i j}=\frac{1}{2}\left(\frac{\partial u_{i}}{\partial x_{j}}+\frac{\partial u_{j}}{\partial x_{i}}\right), \quad u_{i}=u_{i}^{(0)}, \quad x_{k} \in v_{u}, \quad \varepsilon_{i j}=e_{i j}+c_{i j}, \quad e_{i j}=\frac{1+v}{E} \sigma_{i j}-\frac{v}{E} \sigma_{k k} \delta_{i j}+\alpha \Delta T \delta_{i j}, \\
\frac{\partial c_{i j}}{\partial t}=\frac{3}{2} \frac{\dot{c}_{e}}{\sigma_{e}}\left(\sigma_{i j}-\frac{1}{3} \sigma_{k k} \delta_{i j}\right), \quad c_{i j}\left(0, x_{k}\right)=0, \quad \frac{\partial \omega_{c}}{\partial t}=\dot{\omega}_{c}\left(\sigma_{e}, \omega_{c} ; T\right), \quad \omega_{c}\left(0, x_{k}\right)=0, \\
\frac{\partial \omega_{S C C}}{\partial t}=\dot{\omega}_{S C C}\left(\omega_{S C C} ; \sigma_{S C C}, T, \chi_{1}, \chi_{2}, \ldots\right), \quad \sigma_{S C C}=\sigma_{S C C}\left(\sigma_{i j}\right), \quad \omega_{S C C}\left(0, x_{i}\right)=0,
\end{gathered}
$$

where $E$ is the Young modulus of the material, $v$ is its Poisson's ratio, $\alpha$ is the coefficient of thermal expansion, $n_{i}$ are the components of the vector of outer unit normal to the surface of the body $\delta_{i j}$ is the Kronecker symbol, $\Delta T$ is an increment of temperature, $\dot{c}_{e}=\dot{c}_{e}\left(c_{e}, \sigma_{e}, \omega_{c} ; T\right)$ and $\dot{\omega}_{c}\left(\sigma_{e}, \omega_{c} ; T\right)$ are the rates of changes in the equivalent level of creep strains $c_{e}$ and the degree of damage $\omega_{c}$, respectively, depending on the equivalent creep strains and stresses $\sigma_{c}$ and established according to the experimental curves of creep and long-term strength up to fracture at given temperature, $p_{S C C}$ is the pressure of the corrosive medium upon the surface $v_{S C C} \subset v$ with normal $n_{i}$ and unit vectors $\tau_{i}$ and $b_{i}$ lying in the plane tangential to this surface, and $\dot{\omega}_{S C C}=\dot{\omega}_{S C C}\left(\omega_{S C C} ; \sigma_{S C C}, T, \chi_{1}, \chi_{2}, \ldots\right)$ is the law of changes in the parameter of corrosion cracking with time determined according to the experimental data on the corrosion cracking of the material of the body for given temperature, equivalent cracking stress $\sigma_{S C C}$, and the parameters of the medium $\chi_{1}, \chi_{2}, \ldots$.

Equation (3) equipped with initial condition describes the process of growth of corrosion cracks under the conditions of creep depending on the equivalent stress of corrosion cracking $\sigma_{S C C}$ [3]:

$$
\sigma_{S C C}\left(\sigma_{i j}\right)=\max \{\sigma\}, \quad\left|\begin{array}{cc}
\sigma_{i j} \tau_{i} \tau_{j}-\sigma & \sigma_{i j} b_{i} \tau_{j} \\
\sigma_{i j} b_{i} \tau_{j} & \sigma_{i j} b_{i} b_{j}-\sigma
\end{array}\right|=0, \quad x_{k} \in v_{S C C} .
$$


The values $\omega_{S C C}=0$ and $\omega_{S C C}=1$ correspond to the initial (latent) and visible cracks, respectively. The prediction of fracture caused by creep or corrosion cracking is reduced to finding the time $t_{*}$ of termination of latent fracture or the time of appearance of a visible defect at a certain point of the body provided that one of the following conditions is satisfied:

$$
\omega_{c}\left(t_{*}, x_{k}\right)=1 \quad \text { or } \quad \omega_{S C C}\left(t_{*}, x_{k}\right)=1 .
$$

\section{Method for the Solution of the Problem}

The numerical-analytic solution of problem (1)-(5) is constructed on the basis of the Bubnov-Galerkin method described in detail in [5] as applied to the problems of the theory of creep. We rewrite Eqs. (1)-(3) for the main unknown quantities of the posed problem

$$
\mathbf{v}^{T}=\left(c_{11}, c_{22}, c_{33}, c_{12}, c_{23}, c_{32}, \omega_{c}, \omega_{S C C}\right) \quad \text { and } \quad \mathbf{u}^{T}=\left(\sigma_{11}, \sigma_{22}, \sigma_{33}, \sigma_{12}, \sigma_{23}, \sigma_{31}, u_{1}, u_{2}, u_{3}\right)
$$

in the operator form as follows:

$$
\begin{gathered}
\mathbf{A} \cdot \mathbf{u}+\mathbf{C} \cdot \mathbf{v}=\mathbf{f}, \quad x_{k} \in \Upsilon, \\
\frac{\partial \mathbf{v}}{\partial t}=\mathbf{k}(\mathbf{v} ; \mathbf{u}), \quad \mathbf{v}\left(0, x_{k}\right)=\mathbf{0}, \quad x_{k} \in v_{S C C},
\end{gathered}
$$

where $\mathbf{A}, \mathbf{C}$, and $\mathbf{f}$ are, respectively, linear operators and a vector corresponding to the differential equations (1) and $\mathbf{k}$ is an operator corresponding to the differential equations (2) and (3).

According to the Bubnov-Galerkin method, we represent the required solutions in the following approximate form [6]:

$$
\mathbf{v}\left(t, x_{k}\right) \approx \mathbf{V}_{n}\left(x_{k}\right) \cdot \mathbf{v}_{n}(t) ; \quad \mathbf{u}\left(t, x_{k}\right) \approx \mathbf{u}_{v}\left(x_{k}\right)+\mathbf{U}_{n}\left(x_{k}\right) \cdot \mathbf{u}_{n}(t)
$$

where $\mathbf{V}_{n}\left(x_{k}\right)$ and $\mathbf{U}_{n}\left(x_{k}\right)$ are matrices formed by $n$ trial functions, $\mathbf{v}_{n}(t)$ and $\mathbf{u}_{n}(t)$ are vectors formed by the coefficients of approximations $\left[\mathbf{v}_{n}(t)=0\right]$, and $\mathbf{u}_{v}\left(x_{k}\right)$ is a given vector extending the boundary values to the interior of the domain $\Upsilon$.

In approximations (9), we use global trial functions such that the boundary conditions (1) are exactly satisfied for any vector $\mathbf{u}_{n}(t)$. This can be realized by the methods of the theory of $R$-functions, including the domains of noncanonical and complex shape [6].

Further, we substitute approximations (9) in Eqs. (7) and (8). Since the operators $\mathbf{A}$ and $\mathbf{C}$ are linear, the conditions of orthogonality of the discrepancies of equations and trial functions imply that

$$
\mathbf{A}_{n} \cdot \mathbf{u}_{n}+\mathbf{C}_{n} \cdot \mathbf{v}_{n}=\mathbf{f}_{n} \quad \text { and } \quad \frac{d \mathbf{v}_{n}}{d t}=\mathbf{K}_{n}^{-1} \cdot \mathbf{k}_{n}\left(\mathbf{v}_{n} ; \mathbf{u}_{n}\right),
$$

where $\mathbf{A}_{n}, \mathbf{C}_{n}$, and $\mathbf{K}_{n}$ are matrices and $\mathbf{f}_{n}$ and $\mathbf{k}_{n}$ are vectors. 
The matrices $\mathbf{A}_{n}$ and $\mathbf{C}_{n}$ and the vector $\mathbf{f}_{n}$ from the first equation in (10) are given by the formulas

$$
\boldsymbol{A}_{n}=\int_{\Upsilon} \boldsymbol{U}_{n}^{T} \cdot\left(\boldsymbol{A} \cdot \boldsymbol{U}_{n}\right) d \Upsilon, \quad \boldsymbol{C}_{n}=\int_{\Upsilon} \boldsymbol{U}_{n}^{T} \cdot\left(\boldsymbol{C} \cdot \boldsymbol{V}_{n}\right) d \Upsilon, \quad \text { and } \quad \boldsymbol{f}_{n}=\int_{\Upsilon} \boldsymbol{U}_{n}^{T} \cdot\left(\boldsymbol{f}-\boldsymbol{A} \cdot \boldsymbol{u}_{\mathrm{v}}\right) d \Upsilon .
$$

Since Eq. (2) is defined in the entire domain $\Upsilon$ of the body and Eq. (3) is defined only on a part of the boundary surface $v_{S C C} \subset v$, the matrix $\mathbf{K}_{n}$ and the vector $\mathbf{k}_{n}$ have a cellular structure:

$$
\begin{gathered}
\mathbf{K}_{n}=\left(\begin{array}{cc}
\mathbf{K}_{n}^{S C C} & \mathbf{0} \\
\mathbf{0} & \mathbf{K}_{n}^{c}
\end{array}\right), \quad \mathbf{k}_{n}\left(\mathbf{v}_{n} ; \mathbf{u}_{n}\right)=\left(\begin{array}{c}
\mathbf{k}_{n}^{S C C}\left(\mathbf{v}_{n} ; \mathbf{u}_{n}\right) \\
\mathbf{k}_{n}^{c}\left(\mathbf{v}_{n} ; \mathbf{u}_{n}\right)
\end{array}\right), \\
\mathbf{K}_{n}^{S C C}=\int_{v_{S C C}}\left(\left(\mathbf{V}_{n}^{S C C}\right)^{T} \cdot \mathbf{V}_{n}^{S C C}\right) d v, \quad \mathbf{K}_{n}^{c}=\int_{\curlyvee}\left(\left(\mathbf{V}_{n}^{c}\right)^{T} \cdot \mathbf{V}_{n}^{c}\right) d \Upsilon, \\
\mathbf{k}_{n}^{S C C}=\int_{v_{S C C}}\left(\left(\mathbf{V}_{n}^{S C C}\right)^{T} \cdot \mathbf{k}^{S C C}\left(\mathbf{v}_{n} ; \mathbf{u}_{n}\right)\right) d v, \quad \mathbf{k}_{n}^{c}=\int_{\Upsilon}\left(\left(\mathbf{V}_{n}^{c}\right)^{T} \cdot \mathbf{k}^{c}\left(\mathbf{v}_{n} ; \mathbf{u}_{n}\right)\right) d \Upsilon,
\end{gathered}
$$

where $\mathbf{k}^{c}(\mathbf{v} ; \mathbf{u})$ and $\mathbf{k}^{S C C}(\mathbf{v} ; \mathbf{u})$ are vectors corresponding to the right-hand sides of Eqs. (2) and (3), $\mathbf{V}_{n}^{S C C}\left(x_{k}\right)$ is a row matrix of trial functions of the cracking parameter, $\mathbf{V}_{n}^{c}\left(x_{k}\right)$ is a matrix of the trial functions of creep strains and the degree of damage, and $\mathbf{v}_{n}^{S C C}(t)$ and $\mathbf{v}_{n}^{c}(t)$ are vectors of the coefficients of approximation of the cracking parameter and creep strains with the degree of damage.

We exclude the vector $\mathbf{u}_{n}$ from Eqs. (10) and arrive at the following Cauchy-type problem for the vector $\mathbf{v}_{n}:$

$$
\frac{d \mathbf{v}_{n}}{d t}=\mathbf{k}_{n}\left(\mathbf{v}_{n}\right), \quad \mathbf{v}_{n}(0)=\mathbf{0}
$$

where $\mathbf{k}_{n}\left(\mathbf{v}_{n}\right)=\mathbf{K}_{n}^{-1} \cdot \mathbf{k}_{n}\left(\mathbf{v}_{n} ; \mathbf{u}_{n}=\mathbf{A}_{n}^{-1} \cdot\left(\mathbf{f}_{n}-\mathbf{C}_{n} \cdot \mathbf{v}_{n}\right)\right)$.

\section{Numerical Prediction of Corrosion Cracking of the Pipes}

Consider the phenomenon of corrosion cracking of a pipe under the conditions of creep in solutions of magnesium chloride. The pipe is made of 18-8-type stainless steel and its inner and outer radii are equal to $r_{1}$ and $r_{2}$, respectively. In the absence of the axial loads, the pipe is subjected to the action of internal pressure $p$ and has a temperature of $500^{\circ} \mathrm{C}$. The resolving equations used to describe the state of the pipe at points $r_{1} \leq r \leq r_{2}$ are formulated in terms of stresses $\sigma_{r r}=\sigma_{r r}(t, r)$ and $\sigma_{\theta \theta}=\sigma_{\theta \theta}(t, r)$, displacements $u_{r}=u_{r}(t, r)$, creep strains $c_{r r}=c_{r r}(t, r)$ and $c_{\theta \theta}=c_{\theta \theta}(t, r)$, and the degrees of damage $\omega_{c}=\omega_{c}(t, r)$ and corrosion cracking $\omega_{S C C}=$ $\omega_{S C C}(t)$. In view of the axial symmetry of the problem, Eqs. (1)-(3) take the following form in a cylindrical coordinate system: 


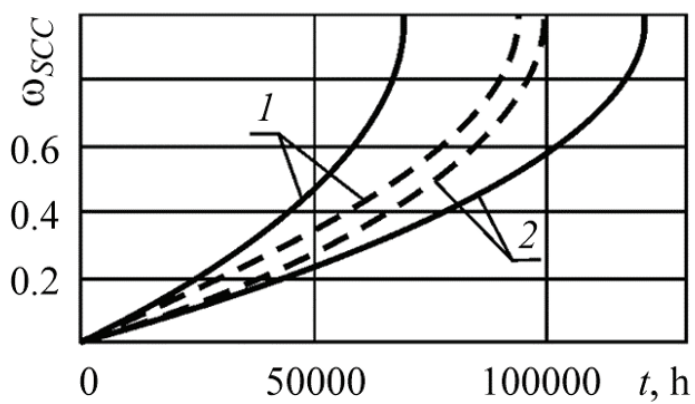

Fig. 1. Dependences of the parameter of corrosion cracking $\omega_{S C C}$ on time $t$ under the action of corrosive media on the inner (curves 1) and outer (curves 2) surfaces of the pipe under the conditions of elastic deformation (solid lines) and creep (dashed lines).

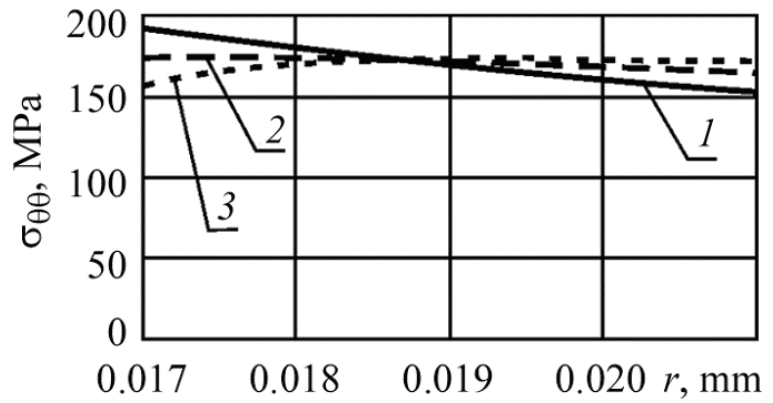

Fig. 2. Distributions of stresses $\sigma_{\theta \theta}$ over the thickness of pipe under the action of internal pressure in the cases of elastic deformation (curve 1) and creep at times $t=28,672 \mathrm{~h}$ (curve 2) and $t \cong 94,637 \mathrm{~h}$ (curve 3).

$$
\begin{gathered}
\frac{\partial \sigma_{r r}}{\partial r}+\frac{\sigma_{r r}-\sigma_{\theta \theta}}{r}=0, \quad \sigma_{r r}\left(t, r_{1}\right)=p, \quad \sigma_{r r}\left(t, r_{2}\right)=0, \\
-\frac{1}{E} \sigma_{r r}+\frac{\nu}{E} \sigma_{\theta \theta}+\frac{\partial u_{r}}{\partial r}-\alpha \Delta T-c_{r r}=0, \quad-\frac{1}{E} \sigma_{\theta \theta}+\frac{v}{E} \sigma_{r r}+\frac{u_{r}}{r}-\alpha \Delta T-c_{\theta \theta}=0, \\
\frac{\partial c_{r r}}{\partial t}=\frac{3}{2} \frac{\dot{c}_{e}}{\sigma_{e}}\left(\frac{2}{3} \sigma_{r r}-\frac{1}{3} \sigma_{\theta \theta}\right), \quad \frac{\partial c_{\theta \theta}}{\partial t}=\frac{3}{2} \frac{\dot{c}_{e}}{\sigma_{e}}\left(\frac{2}{3} \sigma_{\theta \theta}-\frac{1}{3} \sigma_{r r}\right), \quad c_{r r}(0, r)=0, \quad c_{\theta \theta}(0, r)=0, \\
\dot{c}_{e}=B\left(\frac{\sigma_{e}}{1-\omega_{c}}\right)^{q}, \quad \dot{\omega}_{c}=A\left(\frac{\sigma_{e}}{1-\omega_{c}}\right)^{k}, \\
\frac{\partial \omega_{S C C}}{\partial t}=a \cdot 10^{b \sigma_{S C C}+c \chi}\left(1-\omega_{S C C}\right)^{d}, \quad \sigma_{S C C}(t)=\frac{1}{\sqrt{2}} \sqrt{\left(\sigma_{\theta \theta}-\sigma_{r r}\right)^{2}+\sigma_{r r}^{2}+\sigma_{\theta \theta}^{2}},
\end{gathered}
$$

where $E=1.62 \cdot 10^{5} \mathrm{MPa}, \quad v=0.3$, and $\alpha=18.4 \cdot 10^{-6} 1 /{ }^{\circ} \mathrm{C}$ are, respectively, the Young modulus, Poisson's ratio, and the coefficient of linear thermal expansion of 18-8-type stainless steel at $500^{\circ} \mathrm{C}$ [7]. The values 
of the quantities $A=3.799 \cdot 10^{-35} \mathrm{MPa}^{-k} / \mathrm{h}, \quad B=8.859 \cdot 10^{-13} \mathrm{MPa}^{-q} / \mathrm{h}, \quad q=2.023$, and $k=12.344$ were obtained by processing the isochronous curves of creep and long-term strength for the analyzed type of steel [7]. The values of constants $a=1.645 \cdot 10^{-7}, b=6.133 \cdot 10^{-3}, c=9.306 \cdot 10^{-2}$, and $d=1$ correspond to the continual model of corrosion cracking of stainless steels in solutions of magnesium chloride proposed in [3]. For the concentrations of magnesium chloride within the range $\chi=5-10 \%$, this model satisfactorily describes the action of corrosive media in the secondary coolant circuits of nuclear power plants with water-moderated watercooled power reactors. Here, $r_{S C C}$ is a coordinate of a point on the surface of the pipe interacting with corrosive media.

In our numerical predictions of corrosion cracking of the pipes under the action of internal pressure, we take $p=40 \mathrm{MPa}, r_{1}=0.017 \mathrm{~m}$, and $r_{2}=0.021 \mathrm{~m}$. The accumulated numerical results show that corrosion cracking occurs after $\cong 69,075-121,518 \mathrm{~h}$ (Fig. 1), i.e., prior to the termination of latent fracture at $\cong 168,066 \mathrm{~h}$, as a result of creep of the pipe under the action of internal pressure. It is shown that, under the action of corrosive media on the inner surface of the pipe $r_{S C C}=r_{1}$ (Fig. 1, curves 1) under the conditions of creep in the case where the stresses vary as functions of time (Fig. 2), the time of cracking is larger (dashed line) than in the case of elastic deformation (solid line). At the same time, for $r_{S C C}=r_{2} \quad$ (Fig. 1, curves 2), the time of cracking under the conditions of creep is smaller (dashed line) than in the case of elastic deformation (solid line).

The effect of creep on the time of corrosion cracking is explained by the fact that the hoop stresses are redistributed for the period of cracking of the pipe, namely, they decrease with time on the inner surface of the pipe and increase on its outer surface (Fig. 2, curves 2 and 3), as compared with the values corresponding to the case of pure elastic deformation (Fig. 2, curve 1).

\section{REFERENCES}

1. M. I. Reznikov and Yu. M. Lipov, Steam Boilers of Thermal Power Plants [in Russian], Énergoizdat, Moscow (1981).

2. O. E. Andreikiv and N. B. Sas, "Evaluation of the period of subcritical growth of a high-temperature creep crack in the wheel of a steam turbine," Fiz.-Khim. Mekh. Mater., 46, No. 3, 16-22 (2010).

3. O. K. Morachkovskii and Yu. V. Romashov, "Continual model of corrosion-crack growth for the evaluation of the service life of structures," Fiz.-Khim. Mekh. Mater., 46, No. 2, 111-116 (2010).

4. G. S. Pisarenko and N. S. Mozharovskii, Equations and Boundary-Value Problems of the Theory of Plasticity and Creep [in Russian], Naukova Dumka, Kiev (1981).

5. O. K. Morachkovskii and Yu. V. Romashov, "On the solution of initial-boundary-value problems in the theory of creep," Prikl. Mekh., 45, No. 10, 33-44 (2009).

6. V.L. Rvachev, Theory of R-Functions and Some Its Applications [in Russian], Naukova Dumka, Kiev (1982).

7. Gosatoménergonadzor SSSR, Norms of the Strength Analysis of the Equipment and Pipelines of Nuclear Power Plants (PNAÉ G-7002-86) [in Russian], Énergoatomizdat, Moscow (1989). 